FOLIA

Amazónica

Revista del Instituto de Investigaciones

de la Amazonía Peruana

\title{
DIVERSIDAD DE MURCIÉLAGOS EN EL INTERFLUVIO NAPO-PUTUMAYO-AMAZONAS, PERÚ
}

\author{
María Claudia RAMOS-RODRÍGUEZ* ${ }^{1 *}$ Emérita Rosabel TIRADO HERRERA², \\ Pedro PÉREZ-PEÑA ${ }^{3}$ \\ 1 SOIL-PLANT SERVIS S.C.R.L., Iquitos. \\ 2 Universidad Nacional de la Amazonía Peruana (UNAP). Facultad de Ciencias Biológicas. Iquitos. \\ 3 Instituto de Investigaciones de la Amazonía Peruana (IIAP), Iquitos. \\ * Correo electrónico: mclaudia.rrodriguez@gmail.com
}

\section{RESUMEN}

Los murciélagos son uno de los grupos más diversos de mamíferos en el neotrópico y sus funciones como bioindicadores resaltan su importancia. Es así, que tuvimos el interés de conocer su diversidad, estado de conservación y amenazas en el interfluvio Napo-Putumayo-Amazonas, un sector aún poco explorado de la Amazonía peruana. Para ello sistematizamos estudios de diversas fuentes de información registrados en el interfluvio. Encontramos 53 especies de murciélagos, siendo la familia Phyllostomidae y subfamilia Stenodermatinae los más representativos. Las especies Artibeus lituratus, Artibeus obscurus, Lophostoma silvicolum, Phyllostomus elongatus, Rhynchonycteris naso y Sturnira tildae se registraron ampliamente en el Putumayo. Los murciélagos frugívoros e insectívoros fueron más diversos que otros grupos de hábitos alimentarios. Se registra a Diclidurus ingens como especie única, en tanto Sturnira ludovici, Artibeus concolor, Cynomops greenhalli, Molossops neglectus y Platyrrhinus angustirostris como especies raras en el área de evaluación. La composición de murciélagos indica en buen estado de conservación, sin embargo, podría verse afectado por las crecientes actividades ilícitas de deforestación. Se requiere la pronta implementación de proyectos compatibles con las potencialidades del área, que garanticen la conservación de estos bosques que son fuente de vida para muchas especies de murciélagos y otras especies de fauna silvestre.

PALABRAS CLAVE: bioindicadores, Phyllotosmidae, Diclidurus ingens, murciélagos frugívoros. 


\title{
BATS DIVERSITY IN THE NAPO-PUTUMAYO-AMAZONAS INTERFLUVIUM, PERU
}

\begin{abstract}
Bats are one of the most diverse groups of mammals in the neotropics and their functions as bioindicators underscore their importance. Thus, we studied population diversity, conservation status and threats at the Napo-PutumayoAmazonas interfluvium, a sector that is still little explored in the Peruvian Amazon. We systematized studies from various sources focusing on the interfluvium. We found 53 species of bats, with the Phyllostomidae family and sub family Stenodermatinae sub family being the most represented. The species Artibeus lituratus, Artibeus obscurus, Lophostoma silvicolum, Phyllostomus elongatus, Rhynchonycteris naso and Sturnira tildae were widely recorded in Putumayo. There was greater diversity of frugivorous and insectivorous bats than bats of other eating habits. Diclidurus ingens is registered as a unique species, while Sturnira ludovici, Artibeus concolor, Cynomops greenhalli, Molossops neglectus and Platyrrhinus angustirostris are rare species. Given that these forests that are a source of life for many bat and other wildlife species, the urgent implementation of forest conservation projects that are compatible with the potentialities of the area are required.
\end{abstract}

KEYWORDS: bioindicators, Phyllotosmidae, Diclidurus ingens, frugivorous bats. 


\section{INTRODUCCIÓN}

En el interfluvio Napo-Putumayo-Amazonas se realizaron diversos estudios de mamíferos (Pitman et al., 2004; Alverson et al., 2008; Pitman et al., 2011; Pérez-Peña et al., 2019 a,b), incluyendo a los murciélagos, por ser un grupo significativamente con alta riqueza de especies. Estos estudios también han priorizado mamíferos medianos y grandes, sustentados en su importancia como indicadores del estado de conservación, uso por las comunidades rurales y urbanas, así como amenazas antropogénicas que podrían diezmar sus poblaciones. Ante ello, los murciélagos cobran relevancia por ser indicadores del estado de conservación de hábitats (Wilson et al., 1996; Ramos-Rodríguez \& Cevillano, 2019). Además de ser un grupo poco explorado y tener especies aún no descritas en este sector del Perú (Ramos-Rodríguez et al., 2017).

Por otro lado, las creencias y mitos existentes sobre los murciélagos han ocultado los múltiples beneficios que aportan a la salud de los ecosistemas y al bienestar humano (Voigt \& Kingston, 2016). Apenas tres de 99 especies de murciélagos en el departamento de Loreto (Ramos-Rodríguez et al., 2017) son de hábito hematófago (Desmodus rotundus, Diphylla ecaudata y Diaemus youngi) asociados a zonas ganaderas, avícolas y ecosistemas boscosos con presencia de mamíferos medianos y grandes (Quintana \& Pacheco, 2007), mientras que las 96 especies restantes cumplen funciones de regeneración natural de especies pioneras y de sucesiones tardías, controladores de plagas, vertebrados y polinizadores específicos; por tanto es necesario destacar la importancia de estas especies y unificar esfuerzos para seguir contribuyendo a su conocimiento.

Los murciélagos forman parte de la exuberante diversidad de fauna silvestre en el interfluvio Napo-Putumayo-Amazonas; sector que se encuentra categorizado en buen estado de conservación según estudios de composición de especies y estado poblacional de mamíferos medianos y grandes (Pérez-Peña et al., 2019 a,b), lo cual genera expectativa en los grupos más pequeños como los murciélagos. Es este sector se registraron estudios por Montenegro \& Escobedo (2004), Bravo \& Borman (2008), Montenegro \& Moya (2011), asimismo en los sectores fronterizos colombianos y ecuatorianos se encuentran los estudios de Rodríguez-Posada \& CárdenasGonzález (2012), Ramírez-Chaves et al. (2013), Burneo \& Tirira (2014) y Castro (2016), todos ellos enfocados en listas de especies, distribución, presencia y ausencia, así como nuevos reportes de especies. Sin embargo, aún se requiere un análisis de diversidad y unificación de registros en este sector de la Amazonía peruana.

Por tanto, el propósito de este estudio fue conocer la diversidad de murciélagos, así como evaluar las categorías de amenaza y estado de conservación en el interfluvio Napo-PutumayoAmazonas. El estudio contribuirá con información relevante de estos mamíferos y su aporte en la conservación de los bosques amazónicos.

\section{MATERIAL Y MÉTODOS}

\section{ÁREA DE ESTUDIO}

El estudio se realizó en el interfluvio NapoPutumayo-Amazonas, ubicado al noreste del departamento de Loreto, tiene una superficie de $45927,89 \mathrm{~km}^{2}$, limita por el sur con los distritos de Pebas y Ramón Castilla, al oeste con los distritos de Napo y Torres Causana, al norte con la República del Ecuador, y al este con el río Putumayo como límite fronterizo con la República de Colombia (INEI, 2012). En el área se ejecutaron siete estudios de murciélagos (Figura 1, Tabla 1). 
El área presenta un clima tropical, con temperatura media anual de $26,9^{\circ} \mathrm{C}$ y precipitación de 103,3 pulg/año (Climate-Data, 2019). La vegetación está conformada por bosques de colinas baja y alta, terraza alta y baja, varillal pantanoso, aguajal denso y mixto, vegetación ribereña y comunidades intervenidas (Zárate et al., 2019a, b). La fauna silvestre de la zona se caracteriza por ser una de las más conservadas en la Amazonía peruana, con especies de ungulados y primates grandes que son aprovechados para consumo y comercialización (Pérez-Peña et al., 2019c; Ramos-Rodríguez et al., 2019).
Los ríos Napo y Putumayo tienen sus nacientes al norte del Perú, originando fluctuaciones en temporadas diferentes. La creciente de estos ríos se realiza entre los meses de mayo a julio, mientras que la vaciante entre los meses de noviembre a febrero (SEHINAV, 2018)

\section{COLECTA DE INFORMACIÓN}

Se recopiló información de libros, artículos, tesis de pre y postgrado con ayuda de diversas plataformas de información como el Visor geográfico de publicaciones Amazónicas, ALICIA, GBIF, repositorio de la Universidad Nacional de la Amazonía Peruana (UNAP), repositorio

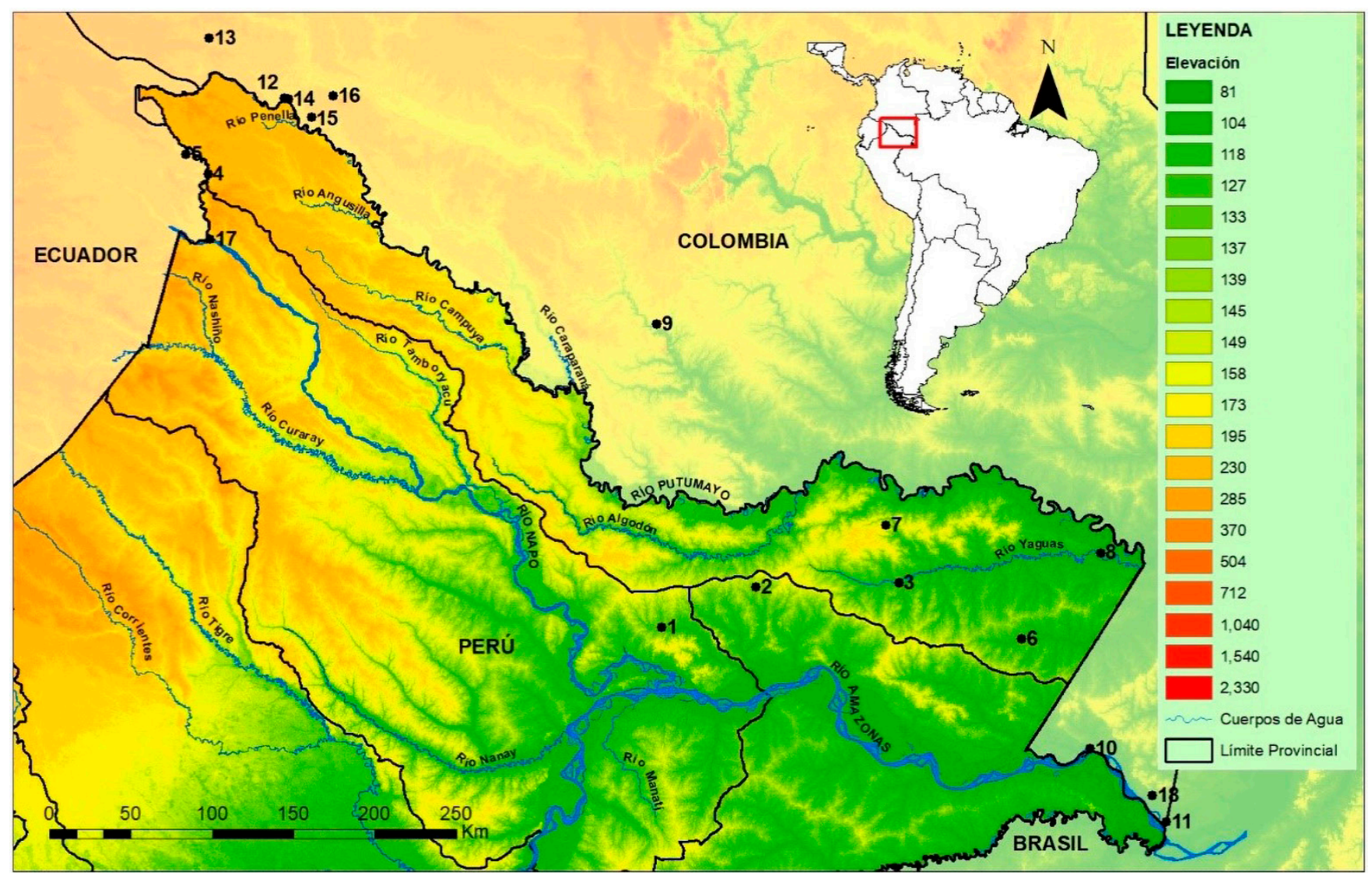

Figura 1: Mapa de ubicación geográfica del Interfluvio Napo-Putumayo-Amazonas. 1= Apayacu, 2= Maronal, 3= Yaguas (Montenegro \& Escobedo, 2004), 4= Redondococha, 5= Garzacocha (Bravo \& Borman, 2008), 6= Alto Cotuhé, 7= Choro, 8= Cachimbo (Montenegro \& Moya, 2011), 9= La Chorrera, 10= Puerto Nariño, 11= Leticia (Rodríguez-Posada \& Cárdenas-González, 2012), 12= Caucaya, 13= Parque Nacional Natural La Paya, 14= Puerto Leguizamo, 15= Gamarra Cesar, 16= La Victoria (Ramírez-Chaves et al., 2013), 17= Napo (Burneo \& Tirira, 2014), 18= Leticia (Castro, 2016). 
Tabla 1. Coordenadas geográficas de los estudios registrados en el interfluvio Napo-Putumayo-Amazonas

\begin{tabular}{|c|c|c|c|c|}
\hline ID & Sector de Evaluación & $x$ & Y & Cita \\
\hline 1 & Apayacu & $-3,116656$ & $-72,722078$ & \multirow{3}{*}{$\begin{array}{l}\text { Montenegro \& } \\
\text { Escobedo, } 2004\end{array}$} \\
\hline 2 & Maronal & $-2,892036$ & $-72,201511$ & \\
\hline 3 & Yaguas & $-2,868419$ & $-71,414083$ & \\
\hline 4 & Redondococha & $-0,602508$ & $-75,225672$ & \multirow{2}{*}{ Bravo \& Borman, 2008} \\
\hline 5 & Garzacocha & $-0,492039$ & $-75,347497$ & \\
\hline 6 & Alto Cotuhé & $-3,179392$ & $-70,7353$ & \multirow{3}{*}{$\begin{array}{c}\text { Montenegro \& Moya, } \\
2011\end{array}$} \\
\hline 7 & Choro & $-2,546969$ & $-71,484528$ & \\
\hline 8 & Cachimbo & $-2,702178$ & $-70,298739$ & \\
\hline 9 & La Chorrera & $-1,433458$ & $-72,749883$ & \multirow{3}{*}{$\begin{array}{c}\text { Rodríguez-Posada \& } \\
\text { Cárdenas-González, } \\
2012\end{array}$} \\
\hline 10 & Puerto Nariño & $-3,788619$ & $-70,355608$ & \\
\hline 11 & Leticia & $-4,191644$ & $-69,934869$ & \\
\hline 12 & Caucaya & $-0,185214$ & $-74,806256$ & \multirow{5}{*}{$\begin{array}{c}\text { Ramírez-Chaves et al. } \\
2013\end{array}$} \\
\hline 13 & $\begin{array}{l}\text { Parque Nacional Natural La } \\
\text { Paya }\end{array}$ & 0,150947 & $-75,218703$ & \\
\hline 14 & Puerto Leguizamo & $-0,186086$ & $-74,783661$ & \\
\hline 15 & Gamarra Cesar & $-0,289819$ & $-74,656325$ & \\
\hline 16 & La Victoria & $-0,168606$ & $-74,533842$ & \\
\hline
\end{tabular}

del Instituto de Investigaciones de la Amazonía Peruana (IIAP) y Google Scholar. La información obtenida fue sistematizada en un archivo Excel, registrándose datos de la especie, coordenadas geográficas, sector de evaluación, método de evaluación, cita y referencia bibliográfica. La nomenclatura fue uniformizada según las referencias de Simmons (2005), Wilson \& Reeder (2005), Gardner (2008) y Solari \& MartíenezArias (2014); las modificaciones del género Dermanura según Solari et al. (2009), Carollia por Solari \& Baker (2006), Zurc \& Velazco (2010), Platyrrhinus por Velazco et al. (2010) y Vampyrodes por Velazco \& Simmons (2011).

\section{DIVERSIDAD}

La diversidad se analizó a través de la riqueza observada y riqueza esperada. La riqueza observada representó el número de especies registradas y la riqueza esperada la cantidad total de especies que habitarían en el área de estudio. Los análisis se realizaron a través de la curva de rarefacción que permite comparar muestras heterogéneas y el estimador no paramétrico Chao 1, para ello se seleccionaron estudios que reporten la abundancia de individuos capturados.

El Análisis de Componentes Principales (ACP) permitió evidenciar la relación de especies con los tipos de bosques, este análisis fue 
complementado con el Análisis de similitud (ANOSIM) que permite evaluar la diferencia de las especies en los sectores estudiados.

\section{ESPECIES ÚNICAS Y RARAS}

Se tuvo en consideración la distribución de los murciélagos en el Perú y Sudamérica, para ello se consideraron las referencias de Simmons (2005), Wilson \& Reeder (2005), Gardner (2008), Noguera-Urbano \& Escalante (2014), LópezBaucells et al. (2014), así como las fuentes de información de GBIF (2020) y UICN (2020).

\section{ESTADO DE CONSERVACIÓN Y AMENAZAS}

El estado de conservación fue evaluado a través de la composición de especies registradas en el área de estudio, teniendo como referente al análisis de diversidad funcional. Además se consideraron estudios con especies indicadoras de hábitats (Galindo-Gonzales, 2004; Ramos-Rodríguez et al., 2018). También se revisó la lista de especies amenazadas consideradas en el Decreto Supremo $\mathrm{N}^{\circ}$ 004-2014-MINAGRI, Libro Rojo de la fauna silvestre amenazada del Perú (SERFOR, 2018) y la Lista roja de la Unión Internacional para la Conservación de la Naturaleza (UICN, 2020). Las amenazas fueron analizadas a través de las referencias de biodiversidad en el área de estudio (Pérez-Peña et al., 2019 a,b) e inventarios rápidos (Pitman et al., 2004; Alverson et al., 2008; Pitman et al., 2011). El análisis conjunto de estas referencias brinda un panorama más amplio de las amenazas en el sector, lo cual permitirá generar propuestas más consensuadas para minimizar los impactos generados.

\section{ANÁLISIS DE DATOS}

La diversidad se analizó con la riqueza observada y esperada. La riqueza se analizó con Chao1 en el software de PAST 3.23 (Hammer, 2019) y el análisis de rarefacción fueron analizados con SigmaPlot 11.0 (Systat Software, Inc., San Jose
California USA). La similitud se realizó con el Análisis de Similitud (ANOSIM) con el índice de Bray Curtis, mientras que el Análisis de Componentes Principales (ACP) permitió evaluar las variables más importantes que explicarían diferencias entre las zonas estudiadas, en ambos casos se usó el software Community Analisis Package 4.0 (Seaby \& Henderson, 2007)

Para la representación gráfica de especies únicas y raras en la región Loreto y en el Perú, se realizó un procesamiento espacial de distribución de especies en función a puntos geográficos registrados en los estudios precedentes y el portal de GBIF.

\section{RESULTADOS}

\section{DIVERSIDAD}

En el interfluvio Napo-Putumayo-Amazonas se registraron 53 especies, diferenciándose un incremento del $64,20 \%$ de especies con respecto al primer estudio desde el 2008 hasta el 2016 (Figura 2).

Las especies de murciélagos estuvieron distribuidas en 11 subfamilias y 5 familias, siendo Phyllostomidae la más representativa $(79,25 \%, 42$ especies) mientras que las familias Emballonuridae (7,55 \%, 4 especies), Molossidae $(7,55 \%, 4$ especies), Noctilionidae $(3,77 \%, 2$ especies), Vespertilionidae $(1,89 \%, 1$ especie) obtuvieron menor a cinco especies (Apéndice 1).

Un total de 27 especies tuvieron un solo registro, entre ellos Artibeus concolor, Artibeus anderseni, Chiroderma salvini, Chiroderma villosum, Cynomops greenhalli, Diclidurus ingens, Diphylla ecaudata, Gardnerycteris crenulatum, Hsunycteris thomasi, Lonchophylla robusta, Lophostoma brasiliense, Micronycteris megalotis, Molossops neglectus, Molossus, Molossus pretiosus, Myotis nigricans, Peropteryx macrotis, 

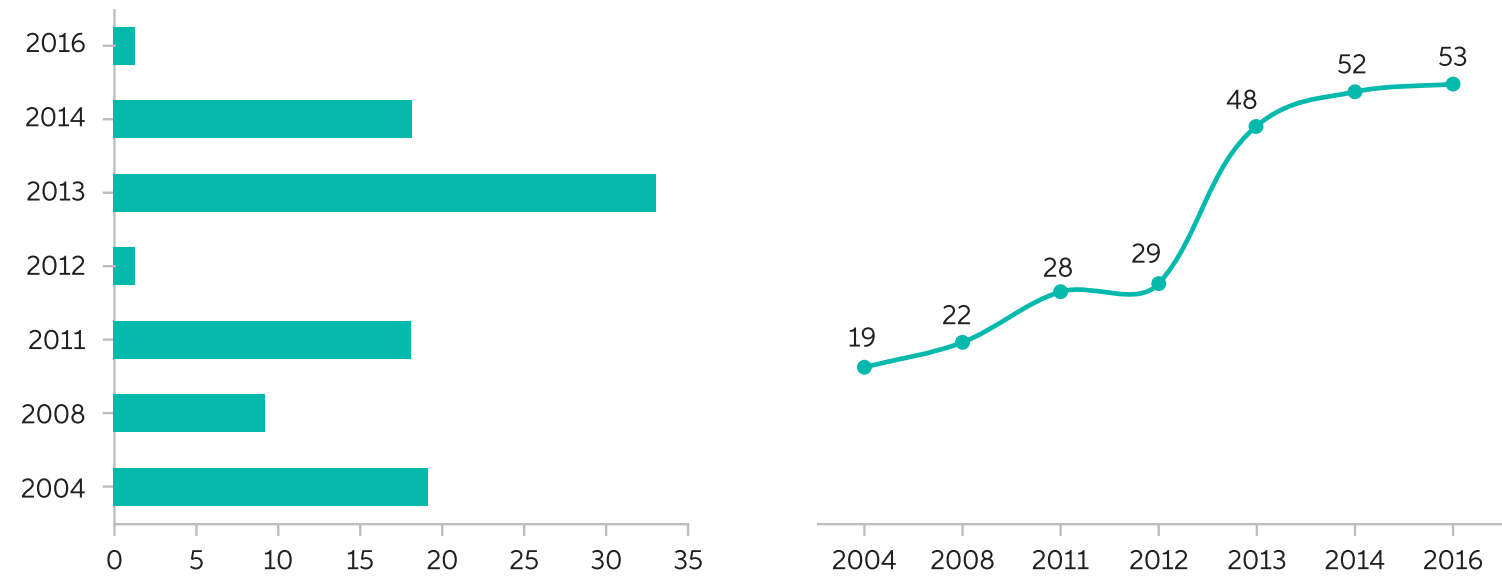

Figura 2: Número de especies de murciélagos registrados desde el año 2004 al 2016 (izquierda) y su acumulación en el interfluvio Napo-Putumayo-Amazonas (derecha).

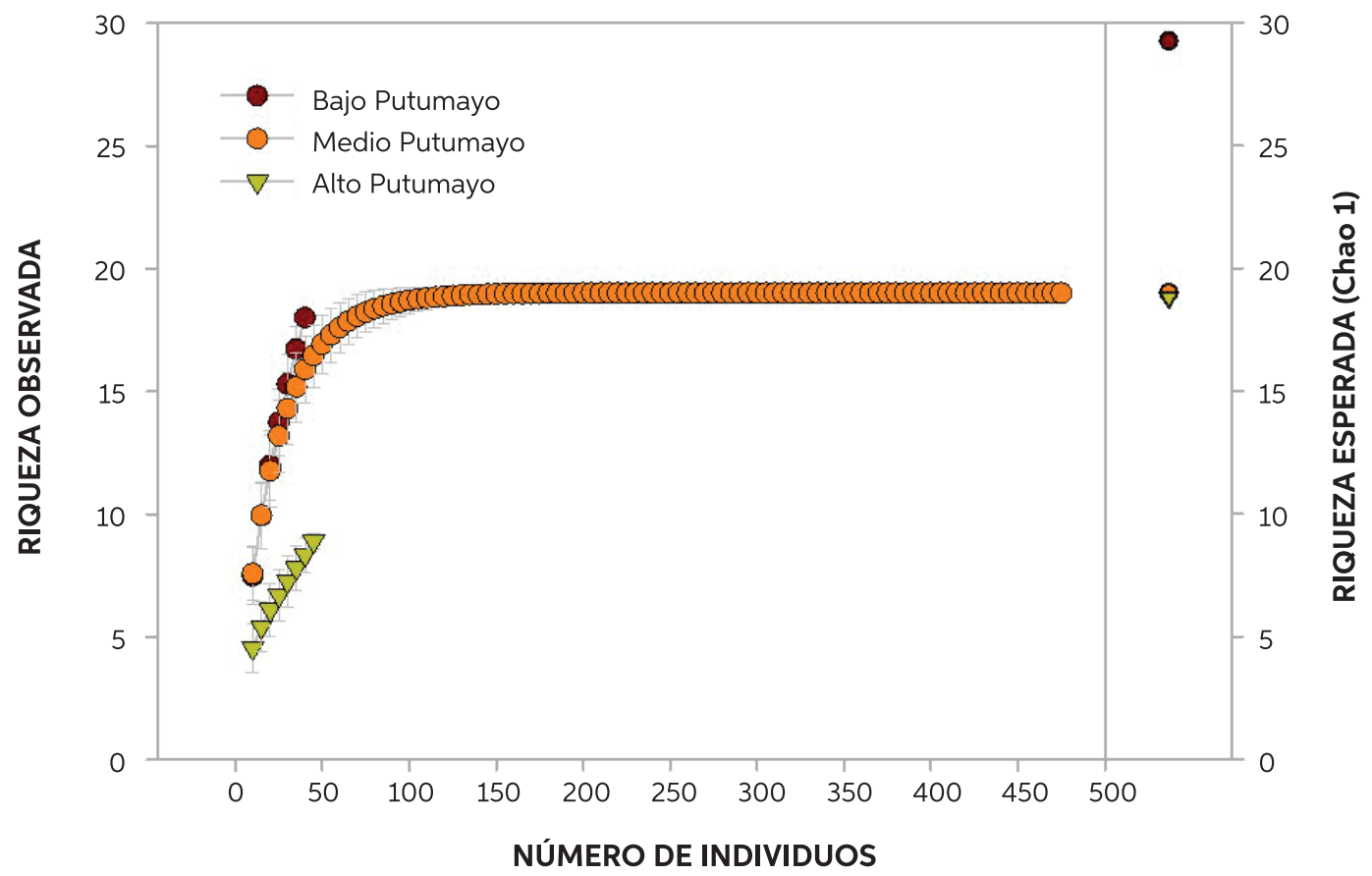

Figura 3. Curvas de rarefacción de la riqueza de especies observadas (izquierda) y esperadas con el estimador Chao1 (derecha) en el bajo, medio y alto Putumayo (Loreto, Perú).

Phylloderma stenops, Platyrrhinus angustirostris, Rhinophylla fischerae, Saccopteryx bilineata, Sturnira aratathomasi, Sturnira bidens, Tonatia saurophila, Uroderma magnirostrum, Vampyriscus bidens y Vampyrodes caraccioli.

La mayor riqueza esperada encontrada corresponde a la cuenca baja del Putumayo con
29 especies (de las 18 especies observadas), mientras que para la cuenca alta fue de 19 especies (de las 5 especies observadas). La riqueza esperada y observada en la cuenca media fue de 19 especies (Figura 3). La mayor cantidad de familias y subfamilias fueron registradas en territorio fronterizo colombiano. El sector 


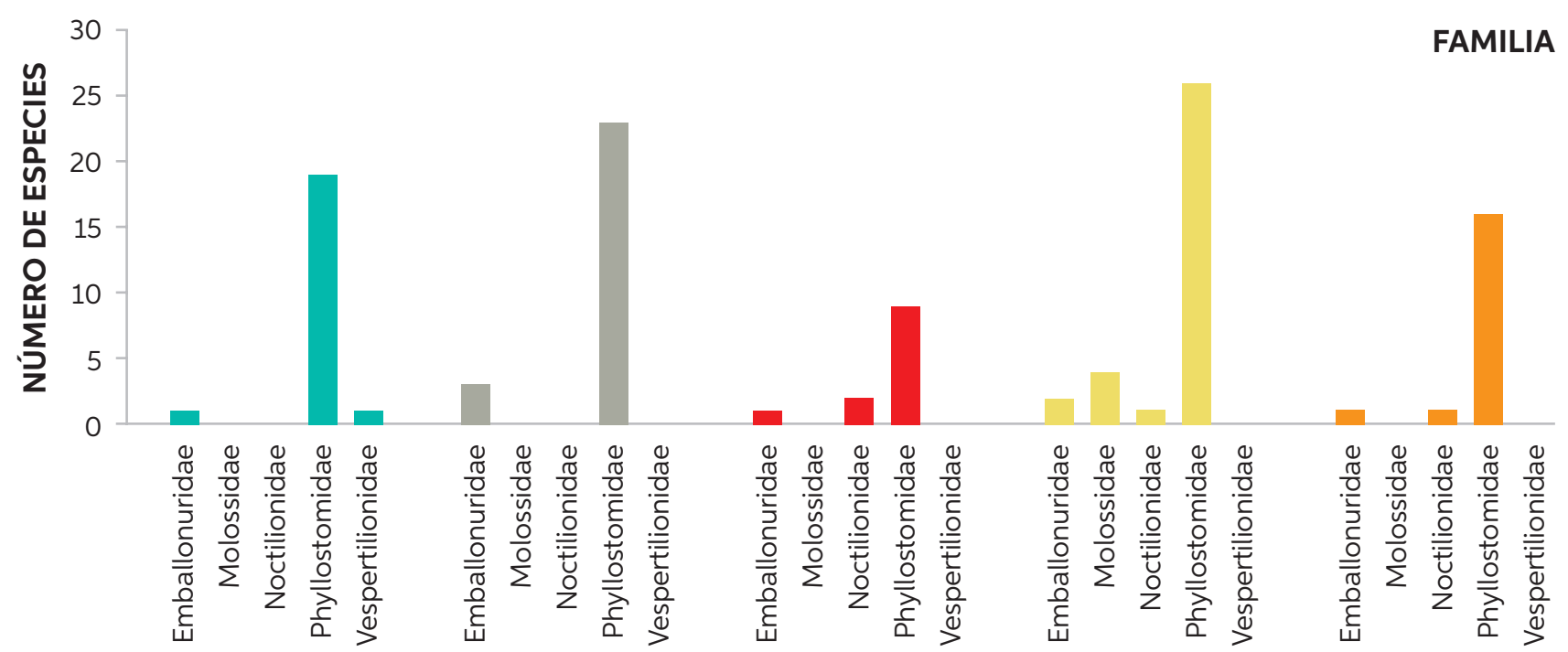

Bajo Putumayo Medio Putumayo

Alto Putumayo

Frontera Colombia

Frontera Ecuador

SUBFAMILIAS

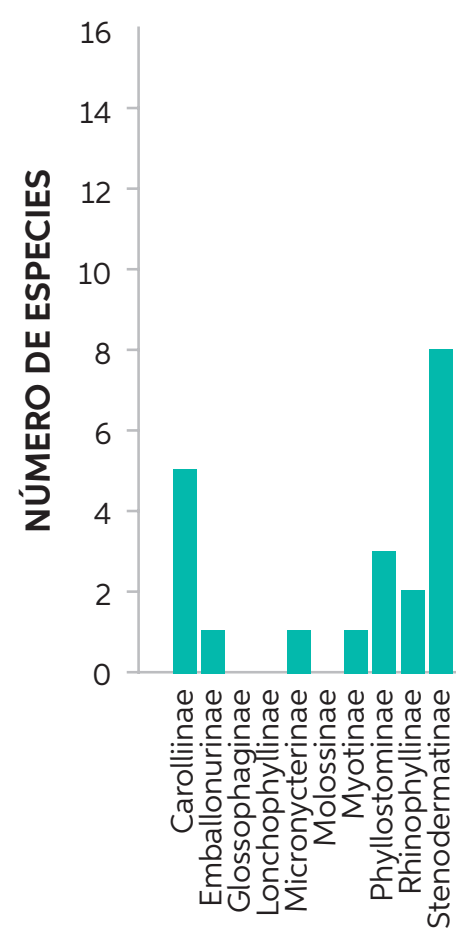

Bajo Putumayo
Medio Putumayo

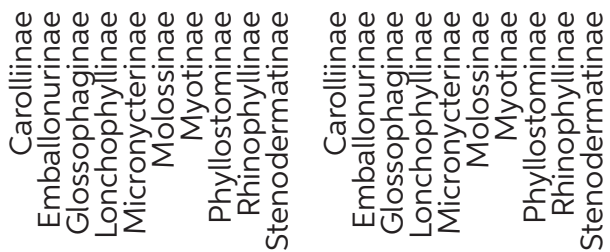

Alto Putumayo

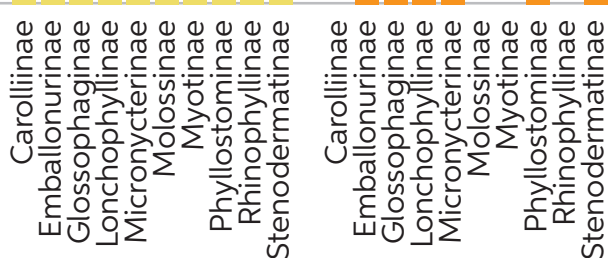

Frontera Colombia

Frontera Ecuador

Figura 4. Riqueza de especies por familia y subfamilia de murciélagos en los en el interfluvio Napo, Putumayo, Amazonas. Nótese la importancia de la familia Phyllostomidae y subfamilia Stenodermatinae. 
con menor cantidad de familias fue el medio Putumayo (Figura 4).

Según el análisis de similitud, los sectores de evaluación resultaron similares (ANOSIM p > 0,05). El Análisis de Componentes Principales explica una variabilidad al 56,03\% en dos componentes principales. El primer componente explica la variabilidad de los murciélagos al 29,52\% donde no se distinguen especies relacionadas a un sector específico, mientras que el segundo componente explica una variabilidad al 26,51\%, donde las especies A. obscurus, C. castanea, C. perspicillata, A. jamaicensis, $S$. ludovici y A. glaucus estuvieron más relacionados a la cuenca media del Putumayo.

\section{ESPECIES ÚNICAS Y RARAS}

Los estudios realizados en el interfluvio NapoPutumayo-Amazonas registran una especie única y cinco especies raras. La especie única corresponde a Diclidurus ingens y las raras a Sturnira ludovici, Artibeus concolor, Cynomops greenhalli, Molossops neglectus, Platyrrhinus angustirostris (Figura 5).

\section{ESTADO DE CONSERVACIÓN Y AMENAZADAS}

El estado de la conservación se evaluó a través de la composición de murciélagos con relación a su diversidad funcional. La riqueza estuvo conformada por mayor cantidad de frugívoros (27 especies, 50,94\%) e insectívoros (16 especies, $30,19 \%$ ), mientras que nectarívoros (4 especies, 7,55 \%), omnívoro (3 especies, 5,66 \%), piscívoro ( 2 especies, 3,77 \%) y hematófago (1 especie, $1,89 \%$ ) estuvieron en menores cantidades (Figura 6), este patrón es una buena referencia de conservación para este grupo de mamíferos.

Dos especies se encuentran en categoría de amenaza, el murciélago cara de perro de Greenhall (Cynomops greenhalli) y el murciélago esbelto de hocico ancho (Platyrrhinus angustirostris), ambos en situación de EN PELIGRO (EN) según el DS 004-2014-MINAGRI y el Libro Rojo de Fauna Silvestre amenazada del Perú. Los estudios lo reportan en el río Putumayo, en Puerto Leguizamo. Ninguna especie se incluyó en las categorías de la UICN y CITES.

En este sector del Perú, la principal amenaza para la comunidad de murciélagos es la deforestación, producto de actividades antropogénicas ilícitas como el cultivo de coca y agricultura migratoria, a ello se suman diversas actividades de extracción de recursos naturales sin medidas de sostenibilidad, tales como tala selectiva, extracción de fauna silvestre, actividad minera y ganadera.

\section{DISCUSIÓN}

Los murciélagos reportados en el interfluvio Napo-Putumayo-Amazonas constituyen un gran aporte al conocimiento de la fauna silvestre en este sector de la Amazonía, con esto se incrementa la riqueza de mamíferos a 123 especies (Montenegro \& Escobedo, 2004; Aquino et al., 2007; Bravo \& Borman, 2008; Vela, 2009; Montenegro \& Moya, 2011; Rodríguez-Posada \& Cárdenas-González, 2012; Ramírez-Chaves et al., 2013; Wong, 2013; Burneo \& Tirira, 2014; Aquino et al., 2016; Bravo et al., 2016; Bowler et al., 2016; Castro, 2016; Ramos-Rodríguez et al., 2019; Pérez-Peña et al., 2019c) donde los murciélagos representan el 43,09\% de esta riqueza.

La buena representatividad de especies va acompañada de diversos beneficios en los ecosistemas del Putumayo, donde el 58,49\% de frugívoras y nectarívoras ayudan en la regeneración natural del bosque a través de la dispersión de semillas y procesos de polinización, mientras que el 41,51 \% cumplen funciones de controladores biológicos debido a la presencia de especies insectívoras, omnívoras, piscívoras y hematófagos. Estos beneficios se 

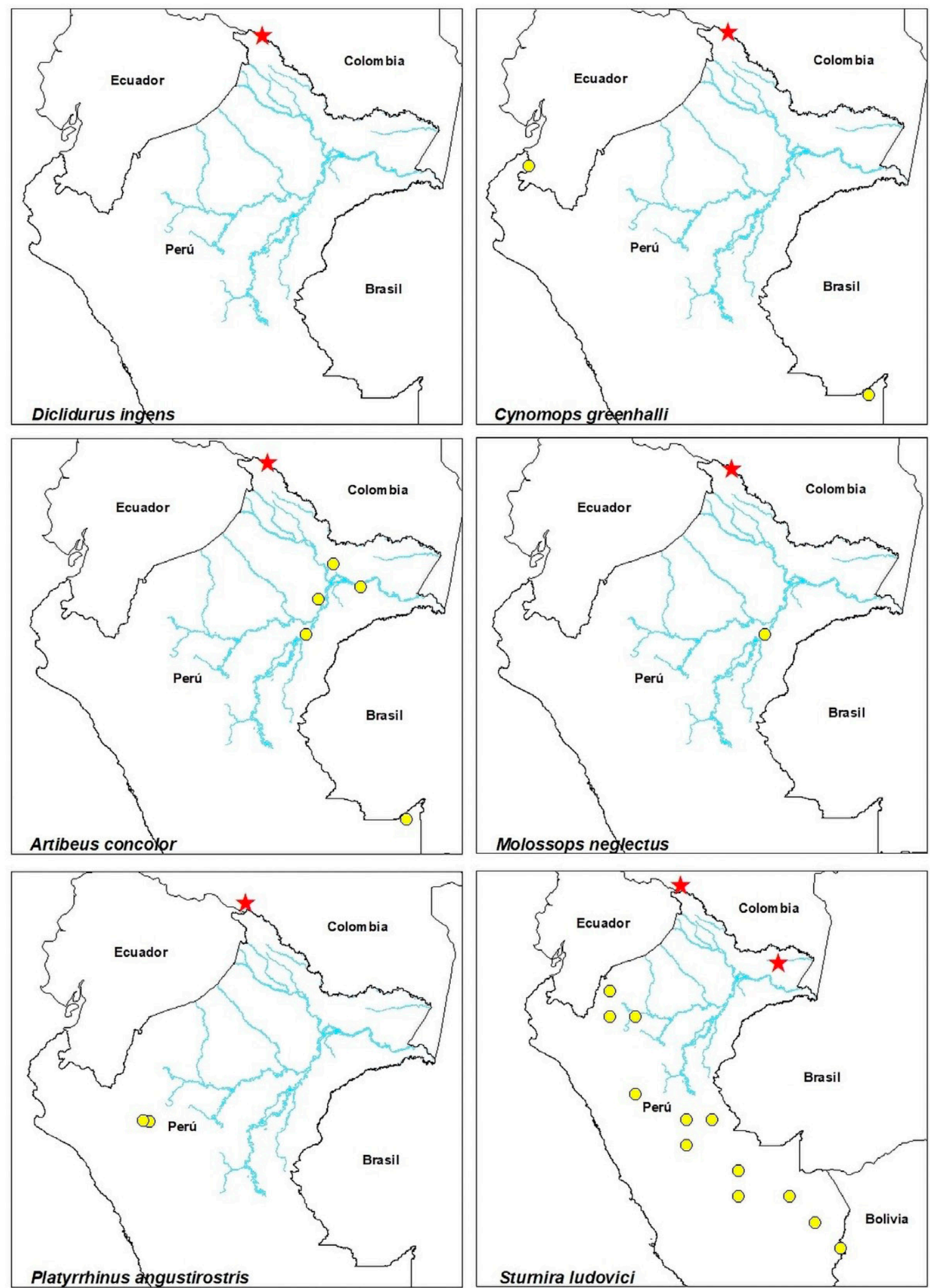

Figura 5. Mapa de especies únicas y raras de murciélagos en el Interfluvio Napo-Putumayo-Amazonas. 


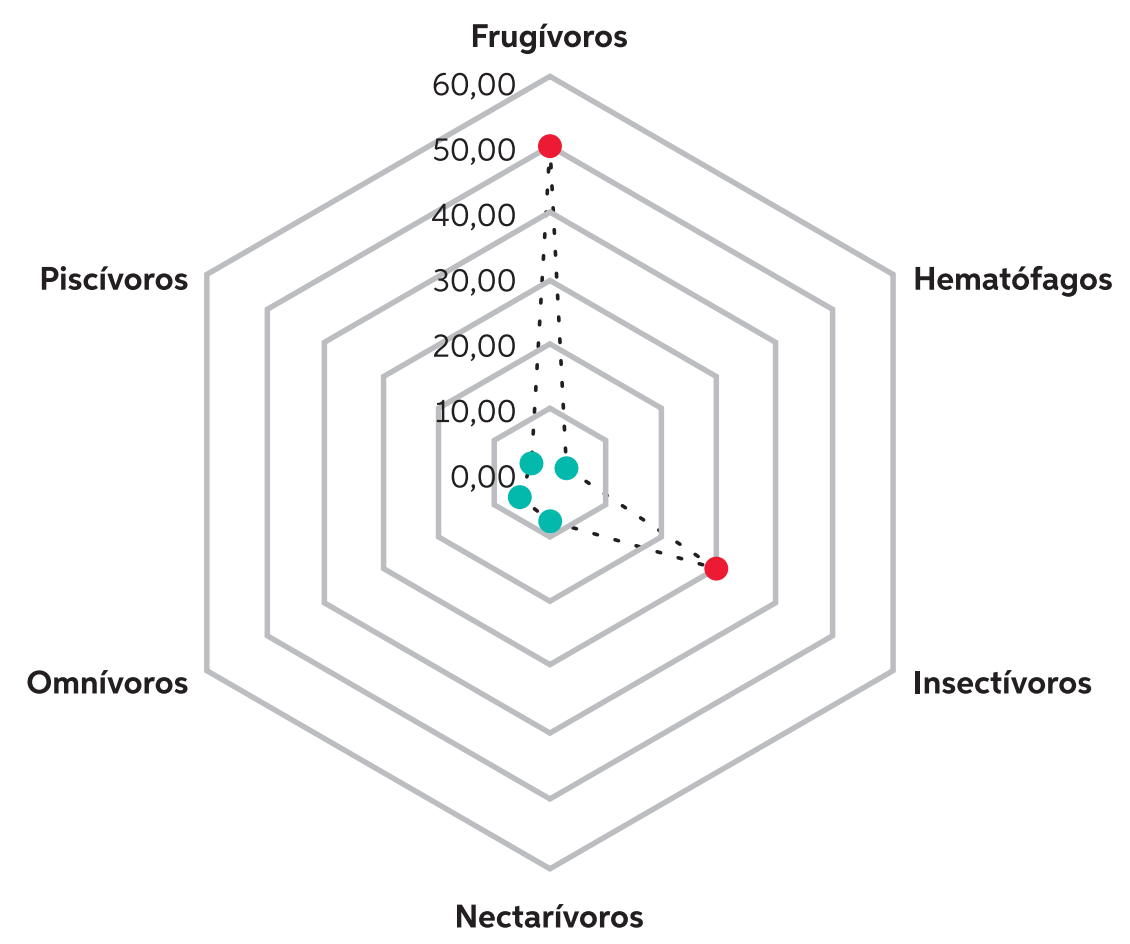

Figura 6. Riqueza funcional de murciélagos en el interfluvio Napo-Putumayo-Amazonas.

han visto subestimados por décadas por ser poco visibles para el hombre. Es así que aún queda un arduo trabajo de investigación para un mejor entendimiento de la dinámica poblacional de murciélagos y sus beneficios a través de los servicios ecosistémicos que brindan a través de sus diversos hábitos alimentarios.

Las especies $A$. lituratus, $A$. obscurus, $L$. silvicolum, $P$. elongatus, $R$. naso y $S$. tildae fueron registradas en todos los estudios de la cuenca baja, media y alta del Putumayo por lo que tendrían una amplia distribución en el área de estudio. El género Artibeus (Phyllostomidae: Stenodermatinae) abarca 20 especies en el neotrópico (Kalko \& Handley, 1994; Simmons, 2005) y su alimentación muestra un mayor consumo de plantas de Ficus (López, 1996; Lou \& Yurrita, 2005; Lobova et al., 2009; Ramos \& Cevillano, 2019; Tirado \& Huerta, en prensa), mientras que el género Sturnira, consume las plantas de los géneros Anthurium, Philodendron y Asplundia (Delaval, 2004; Ramos \& Cevillano, 2019). No se conoce mucho sobre las dietas de los murciélagos por lo que es necesario implementar investigaciones relacionadas a la dieta alimentaria de estas especies en esta área de estudio.

Ambos géneros Artibeus y Sturnira son adaptables a áreas perturbadas (GalindoGonzález, 2004), toleran la transformación de ambientes pudiendo ser vegetación riparia, secundaria y pastizales, consumiendo principalmente las plantas de los géneros Piper, Cecropia y Solanum (Galindo-González, 1998; Ramos \& Cevillano, 2019).

El género Lophostoma prefiere hábitats sin perturbación, aunque suele forrajear en vegetación secundaria, algo similar ocurre con Phyllostomus que prefiere áreas conservadas, pero utiliza la vegetación riparia y corredores para atravesar pastizales sin salir de su protección hacia campo abierto (Galindo-González, 2004), 
mientras que Rhynchonycteris habita áreas conservadas, bosques secundarios y galerías siempre asociado a cuerpos de agua (Tirira, 2017).

El área de estudio presenta una riqueza observada con gran número de registros únicos $(50,94 \%)$, inferimos que este resultado es propio del diseño de muestreo aplicado en los inventarios rápidos, con gran incidencia en la riqueza de especies. El estudio mostró similitud de especies a nivel de cuencas baja, media y alta, consideramos que es necesario aplicar mayor esfuerzo de muestreo por tipo de bosque, lo cual no ha sido especificado en los estudios realizados en el área evaluada. En contraste, la mayor riqueza esperada se evidenció en la cuenca baja del Putumayo, donde se diferencia una gran área inundable, mayor a la cuenca media y alta, quizá la composición de hábitats, vegetación y el buen estado de conservación de este sector podrían influir en la riqueza de especies.

La familia Phyllostomidae tiene mayor diversificación adaptativa entre los vertebrados (Jimenez-Ortega, 2013), además de ser un grupo utilizado como bioindicador en procesos de deforestación (Fenton et al., 1992; Moreno et al., 2005; Mena, 2010; Ramos-Rodríguez et al., 2018). El Putumayo tiene un gran número de especies de esta familia, donde evaluaciones poblacionales podrían resultar efectivas ante perturbaciones antropogénicas frecuentemente realizadas en este sector.

Los estudios del interfluvio Napo-PutumayoAmazonas registraron una especie única, Diclidurus ingens, un Emballonuridae, capturado con un individuo al margen izquierdo de la cuenca alta del río Putumayo, Puerto Leguizamo (Hernandez-Camacho, 1995); este hallazgo es un indicativo que la especie estaría también distribuido en territorio peruano. Así también tuvimos reportes de especies raras tales como Sturnira ludovici, reportado en la costa y sierra del Perú (GBIF, 2020), en el río Yaguas, Loreto (Montenegro \& Escobedo, 2004) y en el Parque Nacional Natural La Paya, río Putumayo (RamírezChaves et al., 2013), lo cual permite reafirmar el crecimiento demográfico de esta especie. Otra especie rara fue Artibeus concolor, un Stenodermatinae con escasos reportes en el Perú, registrado en los departamentos de Madre de Dios, Ucayali y Loreto. Las cantidades registradas en los estudios oscilan entre 1 y 3 individuos. En Loreto fue reportado en los ríos Ucayali (Jenaro Herrera), Nanay (Reserva Nacional Allpahuayo Mishana) y Orosa (Estación Biológica Madre Selva), mientras que en el río Putumayo fue reportado en Puerto Leguizamo (RamírezChaves et al., 2013). Cynomops greenhalli, es un Molossidae con escasos reportes en el Perú, se registró en los departamentos de Madre de Dios (Esselstyn, 2017) y Tumbes (Pacheco et al., 2009; Cadenillas, 2010) en alturas de 250-500 m, como único reporte al noreste del Perú fue en Puerto Leguizamo, río Putumayo (Ramírez-Chaves et al., 2013) a una altura de 186 m. Otra especie de Molossidae como registro raro corresponde a Molossops neglectus, con un reporte en el río Ucayali (Millen \& Lim, 2018) y en Puerto Leguizamo, río Putumayo (Ramírez-Chaves et al. 2013). Platyrrhinus angustirostris, fue reportado en el departamento de Amazonas (Velazco et al., 2010) en un área menor a $5000 \mathrm{~km}^{2}$, entre 1097 1295 m.s.n.m., también está reportado en Puerto Leguizamo, río Putumayo (Ramírez-Chaves et al., 2013) a 186 msnm.

Respecto a las amenazas de conservación, tenemos principalmente actividades ilícitas de deforestación y agricultura migratoria; la pérdida de área boscosa debido al cambio de uso de suelo es un gran problema para la conservación, donde las fragmentaciones generan cambios genéticos a largo plazo (Saunders et al., 1991). En poblaciones reproductivas, la deriva genética ocasiona problemas en poblaciones pequeñas al conducir 
a una disminución poblacional ocasionando alteraciones (Galindo-González, 2004). Es así que las actividades que no se realicen bajo un régimen de sostenibilidad podrían repercutir en las poblaciones de murciélagos, y en general en la fauna, flora y vegetación del área de estudio. Ante ello se sugiere implementar alternativas de desarrollo sostenible compatibles con las potencialidades de la zona de estudio.

\section{CONCLUSIÓN}

El interfluvio Napo-Putumayo-Amazonas presenta una gran diversidad de murciélagos, con una riqueza de 53 especies. La familia Phyllostomidae y subfamilia Stenodermatinae fueron las más representativas, donde los frugívoros e insectívoros fueron más representativos. Se registra una especie única y cinco raras que realzan el interés de invertir esfuerzos en el estudio de este grupo de mamíferos, además de la importancia como bioindicadores de conservación. Esta gran diversidad de especies puede verse afectada principalmente por actividades crecientes de deforestación y agricultura migratoria que se realizan indiscriminadamente sin criterios de sostenibilidad y legalidad. Es así, que resulta imprescindible la presencia de alternativas de desarrollo compatibles con las potencialidades del área de estudio, donde la implementación de proyectos de conservación podría ayudar a mantener las poblaciones de especies focales y por ende otros grupos de animales que habitan en el interfluvio Napo-Putumayo-Amazonas.

\section{AGRADECIMIENTOS}

Este trabajo se realizó gracias al proyecto: Expedición Binacional Perú-Colombia: Inventarios de diversidad biológica en el Gran
Putumayo, en el marco del programa presupuestal 144. Nuestros sinceros agradecimientos a todas las instituciones que realizaron los estudios en esta zona y a los pobladores locales que ayudaron en la ejecución de la misma.

\section{REFERENCIAS BIBLIOGRÁFICAS}

Alverson, W.S.; Vriesendorp, C.; del Campo, A.; Moskovits, D.K.; Stotz, D.F.; García, M.D.; Barbor, L.A. 2008. Ecuador, Perú: CuyabenoGüeppí. Rapid biological and social inventories. Report 20. The Field Museum, Chicago. 376pp. Aquino, R.; López, L.; Arévalo, I.; Daza J. 2016. Diversidad y abundancia de primates y sus amenazas en el interfluvio de los ríos Napo y Putumayo, Amazonía peruana. Revista Peruana de Biología, 23(3): 243-252. DOI: http://dx.doi.org/10.15381/rpb.v23i3.12859

Aquino, R.; Pacheco, T.; Vásquez, M. 2007. Evaluación y valorización económica de la fauna silvestre en el río Algodón, Amazonía peruana. Revista Peruana de Biología, 14(2): 187-192. DOI: https://doi.org/10.15381/ rpb.v14i2.1730

Bowler, M.; Tobler, M.W.; Endress, B.A.; Gilmore, M.P.; Anderson, M. 2016. Estimating mammalian species richness and occupancy in tropical forest canopies with arboreal camera traps. Remote Sensing in Ecology and Conservation. 3(3): 146-157. DOI: https://doi. org/10.1002/rse2.35

Bravo, A.; Borman, R. 2008. Mamíferos. In: Alverson, W.S.; Vriesendorp, C.; del Campo, A.; Moskovits, D.K.; Stotz, D.F.; García, M.; Borbor, L.A. (Eds). Ecuador, Perú: Cuyabeno-Güeppí. Rapid biological and social inventories, Report 20. The Field Museum, Chicago. p. 105-111.

Bravo, A.; Lizcano, D.J.; Álvarez-Loayza, P. 2016. Mamíferos. In: Pitman, N.; Bravo, A.; Claramunt, S.; Vriesendorp, C.; Alvira, D.; Ravikumar, A.; 
del Campo, A.; Stotz, D.F.; Wachter, T.; Heilpern, S.; Rodríguez, B.; Sáenz, A.R.; Chase, Smith R. (Eds). Perú: Medio Putumayo - Algodón. Rapid biological and social inventories, Report 28. The Field Museum, Chicago. p.140-150.

Burneo, S.F.; Tirira, D.G. 2014. Murciélagos del Ecuador: un análisis de sus patrones de riqueza, distribución y aspectos de conservación. Therya, 5(1): 197-228. DOI: https://doi.org/10.12933/therya-14-184

Cadenillas, R.E. 2010. Diversidad, ecología y análisis biogeográfico de los murciélagos del Parque Nacional Cerros de Amotape, Tumbes Perú. Tesis de pre-grado. Universidad Nacional Mayor de San Marcos. Facultad de Ciencias Biológicas, Lima, Perú. 111pp.

Castro, F.F.C. 2016. Nuevo reporte del murciélago hematófago de patas peludas Diphylla ecaudata Spix, 1823 (Chiroptera, Phyllostomidae) en Colombia. Mastozoología neotropical, 23(2): 529-532.

Climate-Data. 2019. El Estrecho. (https:// en.climate-data.org/south-america/peru/ loreto-1045/). Acceso: 05/02/2020.

Decreto Supremo Nº04-2014-MINAGRI. 2014. Decreto Supremo que aprueba la actualización de la lista de clasificación y categorización de las especies amenazadas de fauna silvestre legalmente protegidas. El Peruano. 520497520504.

Delaval, M. 2004. Impacts des perturbations d'origine anthropique sur les peuplements de chauves- souris en Guyane française. Tesis de doctorado. Université de Paris, Paris, Francia. 111pp.

Esselstyn, J. 2017. LSUMZ Mammals Collection. Louisiana State University Museum of Natural Science. Occurrence dataset. (https://doi. org/10.15468/wxiqf6). Acceso: 19/08/2020.

Fenton, M.B.; Acharya, L.; Audet, D.; Hickey, M.B.C.; Merriman, C.; Obrist, M.K. Syme, D.M.; Adkins, B. 1992. Phyllostomid bats
(Chiroptera: Phyllostomidae) as indicators of habitat disruption in the neotropics. Biotropica 24(3): 440-446. DOI: https://doi. org/10.2307/2388615

Galindo-González, J. 1998. Dispersión de semillas por murciélagos: su importancia en la conservación y regeneración del bosque tropical. Acta Zoológica Mexicana (nueva serie), 73: 57-74. DOI: https://doi.org/10.21829/ azm.1998.73731727

Galindo-Gonzales, J. 2004. Clasificación de los murciélagos de la región de los tuxtlas, veracruz, respecto a su respuesta a la fragmentación del hábitat. Acta Zoológica Mexicana, 20(2): 239-243.

Gardner, A.L. 2008. Mammals of South America, volume 1: marsupials, xenarthrans, shrews, and bats. University of Chicago Press. Chicago, Illinois. 669pp.

GBIF. 2020. Global Biodiversity Information Facility. (https://www.gbif.org). Acceso: 19/08/2020.

Hammer, Ø. 2019. PAST. Paleontological STatistic 3.23. (https://www.nhm.uio.no/english/ research/infrastructure/past). Acceso: 19/08/ 2020.

Hernández-Camacho, J. 1995. Una nueva especie colombiana del género Diclidurus (mammalia: chiroptera): Diclidurus ingens. Caldasia, 7(31): 87-98.

INEI. 2012. Encuesta Demográfica y de Salud Familiar. (http://proyectos.inei.gob.pe/web/ biblioineipub/bancopub/Est/Lib1075/index. html). Acceso: 21/08/2020.

Jiménez-Ortega, Á.M. 2013. Conocimiento y conservación de los murciélagos filostómidos (Chiroptera: Phyllostomidae) y su utilidad como bioindicadores de la perturbación de los bosques neotropicales. Tesis de doctorado. Universidad Autónoma de Madrid. Facultad de Ciencias. Madrid. 215pp. 
Kalko, E.V.; Handley, J.C.O. 1994. Evolution, biogeography, and description of a new species of fruit-eating bat, genus Artibeus Leach (1821), from Panamá. Zeitschrift für Säugetierkunde, 59(5): 257-273.

Lobova, T.A.; Geiselman, C.K.; Mori, S.A. 2009. Seed dispersal by bats in the Neotropics. New York Botanical Garden. Nueva York. 465pp.

López,J.1996. Hábitosalimentarios de murciélagos frugívoros en la estación biológica La Selva, Costa Rica. Tesis de Maestría. Universidad Nacional, Sede Heredia. Heredia, Costa Rica. 76pp.

López-Baucells, A.; Rocha, R.; Bobrowiec, P.; Bernard, E.; Palmeirim, J.; Meyer, C. 2016. Field guide to amazonian bats. INPA. Manaos, Brasil. 168pp.

Lou, S.; Yurrita, C. 2005. Análisis de nicho alimentario en la comunidad de murciélagos frugívoros de Yaxhá, Petén, Guatemala. Acta Zoológica Mexicana,21(1):83-94.DOI: https:// doi.org/10.21829/azm.2005.2112009

Mena, J.L. 2010. Respuestas de los murciélagos a la fragmentación del bosque en Pozuzo, Perú. Revista Peruana de Biología, 17:277-284. DOI: https://doi.org/10.15381/rpb.v17i3.2

Millen, B.; Lim, B. 2018. Mammalogy CollectionRoyal Ontario Museum. Version 11.5. Royal Ontario Museum. Occurrence dataset. (https://doi.org/10.15468/2rlrvh). Acceso: 19/08/2020.

Montenegro, O.; Escobedo M. 2004. Mamíferos. In: Pitman, N.; Chase Smith, R.; Vriesendorp, C.; Moskovits, D.; Piana, R.; Knell, G.; Wachter, T. (Eds). Perú: Ampiyacu, Apayacu, Yaguas, Medio Putumayo. Rapid biological and social inventories, Report 12. The Field Museum, Chicago. p. 80-88; 254-261.

Montenegro, O.; Moya L. 2011. Mamíferos. In: Pitman, N.; Vriesendorp, C.; Moskovits, D.K.; von May, R.; Alvira, D.; Wachter, T.; Stotz, D.F.; del Campo, A. (Eds). Perú: Yaguas-Cotuhé.
Rapid biological and social inventories, Report 23. The Field Museum, Chicago. p. 126-133.

Moreno, E.A.; Roa, Y.; Jiménez-Ortega, A.M. 2005. Murciélagos dispersores de semillas en bosques secundarios y áreas cultivadas de la cuenca del río Cabi, Chocó, Colombia. Revista Institucional Universidad Tecnológica del Chocó Diego Luis Córdoba, 23: 45-50.

Noguera-Urbano, E.A.; Escalante, T. 2014 Geographic data for Neotropical bats (Chiroptera). Revista de Biologia Tropical, 62(1): 211-225. DOI: https://doi.org/10.15517/rbt. v62i1.8379

Pacheco, V.; Cadenillas, R.; Salas, E.; Tello, C.; Zeballos, H. 2009. Diversidad y endemismo de los mamíferos del Perú. Revista Peruana de Biología, 16(1):5-32. DOI: https://doi. org/10.15381/rpb.v16i1.111

Pérez-Peña, P.E.; Ramos-Rodríguez, M.C., DíazAlván, J.; Zárate-Gómez, R.; Mejía, K. 2019a. Biodiversidad en la cuenca alta del Putumayo, Perú. Instituto de Investigaciones de la Amazonía Peruana. Iquitos, Perú.154pp.

Pérez-Peña, P.E.; Ramos-Rodríguez, M.C.; DíazAlván, J.; Zárate-Gómez, R.; Mejía, K. 2019b. Biodiversidad en las cuencas del Napo y Curaray, Perú. Instituto de Investigaciones de la Amazonía Peruana; Centro para el Desarrollo del Indígena Amazónico. Iquitos, Perú. 203pp. Pérez-Peña, P.E.; Bardales-Alvites, C.; RamosRodríguez, M.C.; Alcántara, O.E.; Acho, G.W., Lavajos L.E. 2019c. Mamíferos. In: PérezPeña, P.E.; Ramos Rodríguez, M.C.; Díaz Alván, J.; Zárate Gómez, R.; Mejía Carhuanca, K. (Eds). Biodiversidad en las cuencas del Napo y Curaray, Perú. Instituto de Investigaciones de la Amazonía Peruana; Centro para el Desarrollo del Indígena Amazónico. Iquitos, Perú. p. 128-151.

Pitman, N.; Chase-Smith, R.; Vriesendorp, C.; Moskovits, D.; Piana, R.; Knell, G.; Wachter, T. 2004. Perú: Ampiyacu, Apayacu, Yaguas, 
Medio Putumayo. Rapid biological and social inventories, Report 12. The Field Museum, Chicago, Illinois. 273pp.

Pitman, N.; Vriesendorp, C.; Moskovits, D.K.; von May, R.; Alvira, D.; Wachter, T.; Stotz, D.F.; del Campo, A. 2011. Perú: Yaguas-Cotuhé. Rapid biological and social inventories, Report 23. The Field Museum, Chicago, Illinois. 378pp.

Quintana, H.; Pacheco, V. 2007. Identificación y distribución de los murciélagos vampiros del Perú. Revista Peruana de Medicina Experimental y Salud Publica, 24(1): 81-88. DOI: https://doi. org/10.17843/rpmesp.2007.241.1084

Ramírez-Chaves, H.E.; Noguera-Urbano, E.A.; Rodríguez-Posada, M.E. 2013. Mamíferos (Mammalia) del departamento de Putumayo, Colombia. Revista dela Academia Colombiana de Ciencias Exactas, Físicas y Naturales, 37(143): 263-286. DOI: https://doi.org/10.18257/ raccefyn. 9

Ramos-Rodríguez, M.C; Cevillano, S.C.; Aquino, R.; Zárate, G.; Tirado, E. 2017. Diversidad de Murciélagos en bosques de colina de la cuenca alta del Itaya, Loreto, Perú. Folia Amazónica, 26(2): 139-152. DOI: https://doi. org/10.24841/fa.v26i2.430

Ramos-Rodríguez, M.C.; Falcón, R.H.; Díaz, R.E. 2018. Murciélagos indicadores de hábitats perturbados en la Reserva Nacional Allpahuayo Mishana, Perú. Folia Amazónica, 27 (1): 31-46. https://doi.org/10.24841/fa.v27i1.444

Ramos-Rodríguez, M.C.; Cevillano, S.C. 2019. Notas sobre la dieta de murciélagos frugívoros en bosque de colina, Loreto, Perú. Ciencia Amazónica, 7 (1): 79-92. DOI: https://doi. org/10.22386/ca.v7i1.266

Ramos-Rodríguez, M.C.; Pérez-Peña, P.E.; Flores, G.; Ortiz, A. 2019. Mamíferos. In: Pérez-Peña, P.E.; Ramos-Rodríguez, M.C.; Díaz, J.; Zárate, R; Mejía, K. (Eds). Biodiversidad en cuenca alta del Putumayo, Perú. Instituto de Investigaciones de la Amazonía Peruana, Iquitos, Perú. p. 1341511.

Rodríguez-Posada, M.E.; Cárdenas-González, C. 2012. El Murciélago de Visera Sphaeronycteris toxophyllum Peters, 1882 (Chiroptera: Phyllostomidae) en Colombia. Chiroptera Neotropical, 18: 1115-1122.

Saunders, D.A.; Hobbs, R.J.; Margules, C.R. 1991. Biological consequences of ecosystem fragmentation: a review. Conservation biology, 5(1): 18-32. DOI: https://doi. org/10.1111/j.1523-1739.1991.tb00384.x

Seaby, R.M.; Henderson, P.A. 2007. Community Analysis Package version 4.0. (http://www. pisces-conservation.com/softcap.html). Acceso: 21/08/2020.

SEHINAVIF. 2018. Normas técnicas hidrográficas. Levantamientos hidrográficos fluviales. $2^{\mathrm{a}}$ ed. Marina de Guerra del Perú, Lima, Perú. 42pp. SERFOR. 2018. Libro rojo de la fauna silvestre amenazada del Perú. Servicio Nacional Forestal y de Fauna Silvestre - SERFOR. Lima, Perú. 55pp.

Simmons, N.B. 2005. Order Chiroptera. In: Wilson, D.E.; Reeder, D.M. (Eds). Mammal species of the world: a taxonomic and geographic reference. The Johns Hopkins University Press. Baltimore. p. 312-529.

Solari, S.; Martínez-Arias, V. 2014. Cambios recientes en la sistemática y taxonomía de murciélagos neotropicales (Mammalia: Chiroptera). Therya, 5(1): 167-196. DOI: https:// doi.org/10.12933/therya-14-180

Solari, S.; Hoofer, S.R.; Larsen, P.A.; Brown, A.; Bull, R.J.; Guerrero, J.A.; Ortega, J.; Carrera, J.P.; Bradley, R.D.; Baker, R.J. 2009. Operational criteria for genetically defined species: analysis of diversification of the small fruiteating bats, Dermanura (Phyllostomidae: Stenodermatinae). Acta Chiropterologica, 11(2): 279-288. DOI: https://doi. org/10.3161/150811009X485521 
Solari, S.; Baker, R.J. 2006. Mitochondrial DNA sequence, karyotypic, and morphological variation in the Carollia castanea species complex (Chiroptera: Phyllostomidae) with description of a new species. Occasional Papers, Museum of Texas Tech University, 254(14): 1-16.

Tirado, E.R.; Herrera, M.D.J. (en prensa). Importancia de los quirópteros frugívoros en el proceso de germinación de semillas en el Km. 25.3 de la Carretera Iquitos - Nauta. Tesis de pre-grado. Universidad Nacional de la Amazonía Peruana. Facultad de Ciencias Biológicas. Iquitos, Perú. 89pp.

Tirira, D.G. 2017. Guía de campo de los mamíferos del Ecuador. 2 ${ }^{\underline{a}}$ ed. Ediciones Murciélago Blanco. Quito, Ecuador. 600pp.

UICN. 2019. The UICN Red List of Threatened Species. (https://www.iucnredlist.org/ search? query $=$ Carollia $\% 20$ castanea $\&$ searchType $=$ species). Acceso: 15/08/2020.

Vela, C.N. 2009. Determinación de la abundancia de mamíferos en el área de manejo forestal en la comunidad nativa Santa Mercedes, río Putumayo, Loreto. Tesis de pre-grado. Universidad Nacional de la Amazonía Peruana. Iquitos, Perú. 68pp.

Velazco, P.M.; Gardner, A.L.; Patterson, B.D. 2010. Systematics of the Platyrrhinus helleri species complex (Chiroptera: Phyllostomidae), with descriptions of two new species. Zoological Journal of the Linnean Society, 159(3): 785812. DOI: https://doi.org/10.1111/j.10963642.2009.00610.x

Velazco, P.V.; Simmons N.B. 2011. Systematics and taxonomy of great striped-faced bats of the genus Vampyrodes Thomas, 1900 (Chiroptera: Phyllostomidae). American Musseum Novitates, 3710:1-35. DOI: https:// doi.org/10.1206/3710.2
Voigt, C.C.; Kingston, T. 2016. Bats in the anthropocene: conservation of bats in a changing world. Springer Nature. 606pp.

Wilson, E.; Ascorra, C.F.; Solari, S. 1996. Bats as indicators of hábitat disturbance. In: Wilson, E; Sandoval, D. (Eds). Manu, la biodiversidad del sureste de Perú. Smithsonian Institution. Lima, Perú. p. 613-625.

Wilson, D.E.; Reeder, D.M. 2005. Mammal species of the world: a taxonomic and geographic reference. 3a ed. The Johns Hopkins University Press, Baltimore. 2142pp.

Wong, C. 2013. Mamíferos. In: Pitman, N.; Ruelas, E.; Vriesendorp, C.; Stotz, D.F.; Watchter ,T.; del Campo, A.; Alvira, D.; Rodríguez, B., Chase, R.; Sáenz, A.R., Soria, P. (Eds). Perú: Ere Campuya - Algodón. Rapid biological and social inventories, Report 25. The Field Museum, Chicago. p. 121-125.

Zárate, R.; Mori, T.J.; Huaymacari, G.C.; Palacios, J.J.; Pereira, Y.C. 2019a. Vegetación y flora. In: Pérez-Peña, P.E.; Ramos-Rodríguez, M.C.; Díaz Alván, J.; Zárate Gómez, R.; Mejía Carhuanca, K. (Eds). Biodiversidad en la cuenca alta del Putumayo, Perú. Instituto de Investigaciones de la Amazonía Peruana. Iquitos, Perú. p. 1556.

Zárate, R.; Mori, T.J.; Mozombite, L.F.; Palacios, J.J.; Valles, L.A.; \& Huaymacari, G.C. 2019b. Vegetación y flora. In: Pérez-Peña, P.E.; Ramos Rodríguez, M.C.; Díaz Alván, J.; Zárate Gómez, R.; Mejía Carhuanca, K. (Eds). Biodiversidad en las cuencas del Napo y Curaray, Perú. Instituto de Investigaciones de la Amazonía Peruana; Centro para el Desarrollo del Indígena Amazónico. Iquitos, Perú. p.15-52.

Zurc, D.; Velasco, P.M. 2010. Análisis morfológico y morfométrico de Carollia colombiana Cuartas et al. 2001 y C. monohernandezi Muñoz et al. 2004 (Phyllostomidae: Carollinae) en Colombia. Chiroptera Neotropical, 16(1): 567 572. 
Apéndice 1. Riqueza de especies de murciélagos en el interfluvio Napo - Putumayo - Amazonas.

\begin{tabular}{|c|c|c|c|c|c|c|c|}
\hline $\begin{array}{l}\text { Familia } \\
\begin{array}{r}\text { Subfamilia } \\
\text { Especie }\end{array}\end{array}$ & 1 & 2 & 3 & 4 & 5 & 6 & 7 \\
\hline \multicolumn{8}{|l|}{ Emballonuridae } \\
\hline \multicolumn{8}{|l|}{ Emballonurinae } \\
\hline Diclidurus ingens & & & & & $x$ & & \\
\hline Peropteryx macrotis & & & & & & $x$ & \\
\hline Rhynchonycteris naso & $x$ & $x$ & $x$ & & $x$ & & \\
\hline Saccopteryx bilineata & $x$ & & & & & & \\
\hline \multicolumn{8}{|l|}{ Molossidae } \\
\hline \multicolumn{8}{|l|}{ Molossinae } \\
\hline Cynomops greenhalli & & & & & $x$ & & \\
\hline Molossops neglectus & & & & & $x$ & & \\
\hline Molossus molossus & & & & & $x$ & & \\
\hline Molossus pretiosus & & & & & $x$ & & \\
\hline \multicolumn{8}{|l|}{ Noctilionidae } \\
\hline Noctilio albiventris & & & & & $x$ & $x$ & \\
\hline Noctilio leporinus & & $x$ & & & & & \\
\hline \multicolumn{8}{|l|}{ Phyllostomidae } \\
\hline \multicolumn{8}{|l|}{ Carolliinae } \\
\hline Carollia brevicauda & $x$ & & $x$ & & & & \\
\hline Carollia castanea & $x$ & & $x$ & & $x$ & & \\
\hline Carollia perspicillata & $x$ & $x$ & $x$ & & & & \\
\hline \multicolumn{8}{|l|}{ Desmodontinae } \\
\hline Diphylla ecaudata & & & & & & & $x$ \\
\hline \multicolumn{8}{|l|}{ Glossophaginae } \\
\hline Anoura caudifer & $x$ & & & & $x$ & $x$ & \\
\hline Choeroniscus minor & & & & & $x$ & $x$ & \\
\hline \multicolumn{8}{|l|}{ Lonchophyllinae } \\
\hline Hsunycteris thomasi & & & & & $x$ & & \\
\hline Lonchophylla robusta & & & & & & $x$ & \\
\hline \multicolumn{8}{|l|}{ Micronycterinae } \\
\hline Micronycteris megalotis & & & $x$ & & & & \\
\hline Micronycteris minuta & & & & & $x$ & $x$ & \\
\hline \multicolumn{8}{|l|}{ Phyllostominae } \\
\hline Gardnerycteris crenulatum & & $x$ & & & & & \\
\hline Lophostoma brasiliense & & & & & $x$ & & \\
\hline Lophostoma silvicolum & $x$ & $x$ & $x$ & & $x$ & $x$ & \\
\hline
\end{tabular}


Phylloderma stenops

Phyllostomus elongatus

Phyllostomus hastatus

Tonatia saurophila

Trachops cirrhosus

Rhinophyllinae

Rhinophylla fischerae

Rhinophylla pumilio

Stenodermatinae

Artibeus concolor

Artibeus glaucus

Artibeus jamaicensis

Artibeus lituratus

Artibeus obscurus

Artibeus planirostris

Artibeus anderseni

Chiroderma salvini

Chiroderma villosum

Mesophylla macconnelli

Platyrrhinus angustirostris

Sphaeronycteris toxophyllum

Sturnira aratathomasi

Sturnira bidens

Sturnira lilium

Sturnira ludovici

Sturnira magna

Sturnira tildae

Uroderma magnirostrum

Vampyressa thyone

Vampyriscus bidens

Vampyrodes caraccioli

$\begin{array}{ll}x & x \\ x & x \\ x & \end{array}$

X

$X$

$x$

$x$

$x$

$x$

$x \quad x$

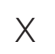

$x$

$x$

$x$

$x$

X

$x \quad \begin{aligned} & x \\ & x \\ & x\end{aligned}$

$x$

$x$

$x$

$x$ $x$

$x$

$x$

$x$

$x$

$x$

$x$

$x$

$x$

$x \quad x$

$x$

$x$

$x$

$x \quad x$

$x \quad x$

$x$

$x$

$x$

$x$

X

$x$

$x$

$x$

$x$

$x$

$x$
$x-10$

X

Vespertilionidae

Myotinae

Myotis nigricans

Total. Especies $=53$

19

9

$x$

18

1

33

18

1

Leyenda. 1= Montenegro \& Escobedo (2004), 2= Bravo \& Borman (2008), 3= Montenegro \& Moya (2011), 4= Rodríguez-

Posada \& Cárdenas-González (2012), 5= Ramírez-Chaves et al. (2013), 6= Burneo \& Tirira (2014), 7= Castro (2016).

Recibido: 22 de setiembre de 2020 Aceptado para publicación: 17 de noviembre de 2020

Esta obra está bajo una Licencia Creative Commons Atribución-NoComercial-SinDerivar 4.0 Internacional. 